\title{
A Knowledge Server for Reasoning about Temporal Constraints Between Classes and Instances of Events*
}

\author{
Paolo Terenziani \\ Dipartimento di Informatica \\ Università del Piemonte Orientale “Amedeo Avogadro” \\ Spalto Marengo 33, 15100 Alessandria, Italy \\ E-mail: terenz@mfn.unipmn.it \\ Luca Anselma \\ Dipartimento di Informatica \\ Università di Torino \\ Corso Svizzera 185, 10149 Torino, Italy \\ E-mail: anselma@di.unito.it
}

\begin{abstract}
Reasoning with temporal constraints is a ubiquitous issue in many computer science tasks, for which many dedicated approaches have been and are being built. In particular, in many areas, including planning, workflow, guidelines and protocol management, one needs to represent and reason with temporal constraints between classes of events (e.g., between the types of actions needed to achieve a goal) and temporal constraints between instances of events (e.g., between the specific actions being executed). The temporal constraints between the classes of events must be inherited by the instances, and the consistency of both types of constraints must be checked. In this paper, we design a general-purpose domain-independent knowledge server dealing with these issues. In particular, we propose a formalism to represent temporal constraints, and we point out two orthogonal parameters that affect the definition of reasoning algorithms operating on them. We then show four algorithms to deal with inheritance and to perform temporal consistency checking (depending on the parameters) and we study their properties. Finally, we report the results we obtained by applying our system to the treatment of temporal constraints in clinical guidelines.
\end{abstract}

Keywords: Knowledge Representation, Temporal reasoning, Temporal Constraints, Inheritance

* This is a preprint of an article accepted for publication in International Journal of Intelligent Systems ( 2004 (copyright owner as specified in the Journal) 


\section{Introduction}

Time plays a fundamental role in the modelling of reality, and reasoning about time is essential in most intelligent activities. For instance, dealing with time is important in many different areas of Computer Science and Artificial Intelligence (henceforth: AI). Therefore, many AI approaches focused their attention to the definition of suitable formalisms to represent time-related phenomena and to reason with them. Roughly speaking, one could distinguish between two different mainstreams in the research about time carried on within the AI community (see [Terenziani, 03b]).

The approaches in the first mainstream mainly focus on the definition of a formalism general enough to represent a wide range of temporal phenomena. Such approaches aim at dealing with the dynamic aspects of the world, at describing the internal structure of actions and events occurring over the world, and at modelling how the world changes in response to such actions. Although very different formalisms have been devised to such a purpose, most of these approaches proposed first order, modal, or nonmonotonic logical formalisms.

On the other hand, the approaches in the second mainstream (henceforth, we will call them "constraintbased" approaches), such as the one we are going to describe in this paper, mainly focus on the definition of representation formalisms and of reasoning techniques to deal specifically with temporal constraints between temporal entities per se, independently of the events and states which take place over such entities. For instance, given three time intervals I1, I2 and I3, if I1 is before I2 and I2 is before I3, then one can infer that I1 is before I3, independently of the events that occurred in I1, I2 and I3. By focusing on a more restricted problem, one can obtain better results than general-purpose approaches. For instance, with a careful definition of the temporal constraint language, one can define specialised reasoning techniques that make inferences such as the above one in a more efficient way than, e.g., a standard theorem prover for the first-order logic. As a consequence, the analysis of the trade-off between the expressiveness of the constraint language and the computational complexity of the inference techniques operating on them is a central issue within the constraint-based mainstream of research (see, e.g., the survey in [Vila, 94]). Dealing with temporal constraints is a fundamental task in many AI applications, including planning, scheduling, natural language understanding, protocol and guideline management and, in general, knowledge representation. This fact motivated a large deal of AI research aimed at building application-independent and domain-independent managers of temporal constraints (see, e.g., the special issue [IJIS, 91] and the comparison of different systems in [Allen \& Yampratoom, 93]). Such managers are intended to be specialised knowledge servers that represent and reason with temporal constraints, and that co-operate with other software modules in order to solve problems in different applications (see, e.g., 
[Allen, 91a; Console \& Terenziani, 99]). For instance, in planning problems, a temporal manager could cooperate with a planner, in order to check incrementally the temporal consistency of the plan being built. In general, the adoption of a specialised temporal manager is very advantageous from the computational point of view, and allows programmers to focus on their domain and application specific problems and to design modular architectures for their systems.

In AI constraint-based approaches, special attention was dedicated to the treatment of qualitative temporal constraints (such as, e.g., "before" or "during"; consider, e.g., [Allen, 83] and the survey in [Vilain et al., 90]), quantitative constraints such as dates, durations, and delays (consider, e.g., Dechter et al.'s STP and TCSP [Dechter et al., 91]) and, more recently, to the integration of both types of constraints (see, e.g., [Brusoni et al., 97; Meiri, 91] and the surveys in [Allen, 91a; Vila, 94]). All these approaches dealt with temporal constraints between time points and/or time intervals. In most applications, such points (intervals) were the temporal extension of specific instances of events only (e.g., "yesterday the reservation of patient $\mathrm{X}$ was performed 3 hours before the laboratory test"); on the other hand, in other applications (see, e.g., [Allen, 91b]) points (intervals) represented "classes" of events such as those in protocols, guidelines, schedules, workflows (e.g., "reservation must be performed at least 2 hours before laboratory tests"). However, notice that in no case both temporal constraints on classes and temporal constraints on instances were treated at the same time by these temporal approaches.

However, such an integrated treatment of temporal constraints of classes plus instances is very useful in many areas, such as planning, workflow management, protocol/guideline management and so on. For instance, an event (or action) in a general plan (or workflow, or protocol, or guideline) represents a class (set) of instances of events, in the sense that it has specific instantiations for specific executions of the plan itself. On the other hand, while executing (instantiating) a general plan, one has specific instances of the classes of events in the plan. Such instances take place at times that can be specified in a more or less accurate and precise way (depending on the accuracy of the observation of their time of occurrence) via a set of temporal constraints. Obviously, the instances must respect (i.e., be consistent with) the constraints they inherit from their super-classes. However, such a consistency can only be checked through an integrated treatment of both constraints on classes and constraints on instances in the same reasoning process. Such a treatment involves the analysis of different issues, including the underlying semantics of temporal constraints on classes, temporal constraint inheritance, and the predictive role of constraints on classes (see the next section). These are ubiquitous problems in many AI tasks and applications, so that we believe that they deserve specific attention, and that it is worth to build a task and domain independent knowledge server dealing with them, to isolate and solve once and for all the problem (so 
that sotware developers can focus on the specific problems of their application, and demand temporal constraint reasoning problem to the knowledge server) ${ }^{1}$. However, to the best of our knowledge, no such general-purpose constraint-based knowledge server exist. The goal of the work in this paper is to lay the foundations of the design and development of such a tool.

In the second section, we point out more clearly the issues to be addressed by an integrated constraintbased temporal manager system dealing with both temporal constraints on classes and on instances (for short, in the following, we will call "classes+instances" temporal manager such a tool). We also point out two orthogonal parameters, which underlie the definition of a classes+instances temporal manager: the capability of dealing with (i) future events and/or (ii) complete vs. incomplete observations. In the third section, we first sketch the STP framework ([Dechter et al., 91]), which is a well-known constraint framework at the basis of many temporal managers (see, e.g., [Brusoni et al., 97; Meiri, 91]). Then, we introduce a high-level language to deal with temporal constraints between instances and a language to represent temporal constraints between classes. For the sake of simplicity, both languages cope only with temporal constraints that can be mapped onto the STP framework. Sections 4 ad 5 are the core of the paper, since they describe algorithms for integrated consistency checking, and analyse their correctness and complexity. In particular, in the fourth section, we deal with constraint inheritance and classes+instances temporal reasoning to check consistency in case (1) future events are considered and (2) the observations on instances are not complete. In section five, we cover also the three remaining combinations of the parameters (i) and (ii), extending the approach in section four to cover the cases where observations are complete (i.e., when one is sure that all the events which occurred have been observed) and/or no future event is considered. In the sixth section, we sketch the preliminary results we obtained by applying our domain and task independent temporal knowledge server to temporal reasoning on clinical guidelines. Finally, the seventh section contains concluding remarks.

\footnotetext{
${ }^{1}$ For instance, this is the approach which has been followed, starting from the eighties, in order to deal with the description of classes and instances of entities, and with the automatic classification of entities according to their description. KL-ONE [Brachman \& Schmolze, 85] and KL-ONE-like hybrid knowledge representation systems and their descendants (consider, e.g., Description Logics [Calnavese et al., 98]; see, e.g., the survey in [Nebel, 90]) are probably the most popular examples of task and domain independent knowledge server systems, providing users and other systems with facilities for storing and reasoning with classes (in the so-called T-Box), instances (in the A-Box) and inheritance [Levesque \& Brachman, 87]. This showed to be very advantageous both from the conceptual and from the engineering point of view: instead of having to deal from scratch with classes, instances and inheritance, programmers and knowledge engineers could use such systems to this purpose, and focus on the specific problems of their task/application domain (see, e.g., in [Schmolze \& Mark, 91] a survey of applications).
} 
Classes and Instances of Events. The distinction between classes of events and instances of events (henceforth, we use event as a cover term for agentive and not-agentive actions) is only a specific case of the wellknown distinction between classes and instances. For example, in a general guideline or plan (e.g., in the clinical field), one may represent the event (action) of "performing a laboratory test". Such an event stands for a class (of events), in the sense that it represents a set of individual occurrences of "performing a laboratory test", taking place at definite intervals of time. A specific person may execute, at a given time, a specific laboratory test. This is, of course, an instance event (i.e., a specific occurrence) of the above class of events. This can be graphically represented as in Figure 1, where LT1, LT2, ... LTk represent specific instances (Instance-of arcs) of the event class "Lab_Tests" occurring at specific intervals of time.

Temporal Constraint Inheritance. Usually, general plans (guidelines, protocols, workflows) contain temporal constraints between (classes of) events. For example, in the CLASSES part of Figure 1, we graphically represent in a simplified way part of a guideline for the management of laboratory tests in a hospital. The general guideline represents the fact that the reservation (RS) of each test must be done between 1 and 7 days before the lab-test (LT), and that the results of the tests are reported (RP) within 1 and 48 hours after the end of the test. Of course, these are temporal constraints between classes of events, which might be instantiated many times, for different instantiations of the classes of events (see the INSTANCES part of Figure 1). The binary temporal constraints at the class level are "relational" constraints, in the sense that they have to be inherited only by "corresponding" pairs of instances of the related classes. For example, in Figure 1, the constraint between RS and LT states that each instance of Reservation must be 1-7 days before the corresponding instance of Lab-Test (and not before all LT instances!). In general, a temporal constraint R between two classes C1 and C2 of events involves an underlying relation pairing instances of the two classes. This pairing relation has been recognised, e.g., by [Morris et al., 93; Terenziani, 97]. As in [Morris et al., 93], we call this relation correlation.

In the example in Figure 1, we suppose that RSi is correlated to LTi which, in turn, is correlated to RPi, $1 \leq \mathrm{i} \leq \mathrm{k}$. 


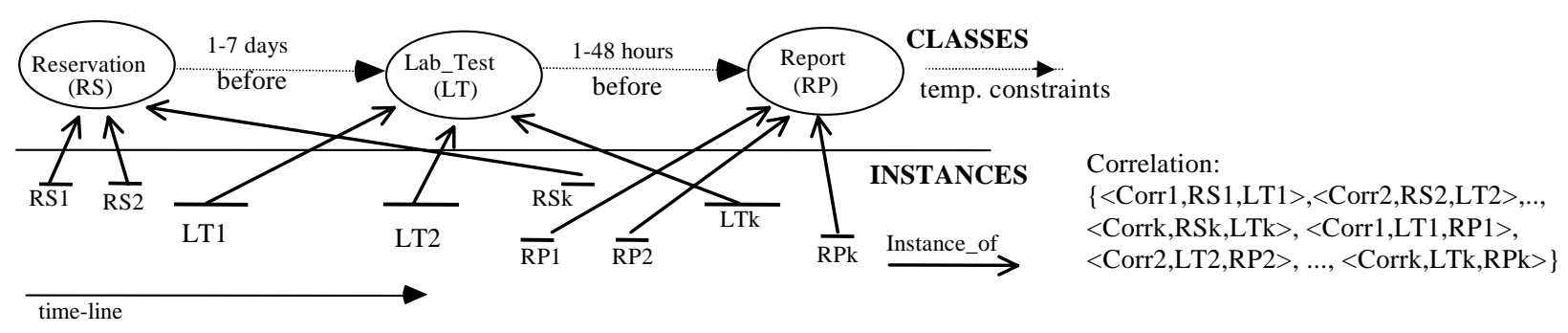

Figure 1 Temporal constraints between classes of events and between instances.

Correlation. Although correlation is at the core of the meaning of temporal constraints between classes of events, it has not a clear characterization in the literature. For instance, Morris [Morris et al., 93] (who studied it in the context of qualitative temporal constraints about periodic events) pointed out that correlation is symmetric and transitive, and simply stated that "correlation is an equivalence relation between pairs of convex parts of recurring events that holds as a result of binary relations holding between them” ([Morris et al., 93, pag. 656]). This difficulty is certainly due to the fact that the nature of correlation depends on the problem and the context. Even in our simple clinical example, correlation may depend on the level of detail used to describe events, and on assumptions on the specific application. E.g., an instance R of Reservation may be correlated to an instance L of Lab_Test if:

(1) Both L and R refer to the same patient code and to the same type of test. However, this rule holds only in case a patient cannot have multiple tests of the same type;

(2) Both L and R refer to the same patient and to the same type of test in the same period. This rule can be used only if a patient cannot have two tests of the same type exactly at the same period of time;

(3) Both L and $\mathrm{R}$ refer to the same "unique code". This rule can be used in the extreme case: the correspondence is given by a code, unique for each execution of a lab test.

Correlation can be represented by a (symmetric and transitive) predicate $\operatorname{COR}(x, y, z)$ representing the fact that $y$ and $z$ are correlated by the correlation $x$, where $y$ and $z$ are instances of events. E.g., in the hypothesis 1 above, $\operatorname{COR}\left(C_{P 1-T 1}, R S 1, L T 1\right), C O R\left(C_{P 1-T 1}, L T 1, R P 1\right)$ may represent the fact that RS1, LT1 and RP1 are pairwise correlated by correlation $\mathrm{C}_{\mathrm{P} 1-\mathrm{T} 1}$ (regarding patient P1 and the type of test T1). Different correlation constants can be used to model, e.g., the fact that patient $\mathrm{P} 1$ also had to make test $\mathrm{T} 2$ (i.e., $C O R\left(C_{P 1-T 2}, R S 2, L T 2\right), C O R\left(C_{P 1-T 2}\right.$, $L T 2, R P 2)$ ) and that also patient $\mathrm{P} 2$ had to make test $\mathrm{T} 2$ (i.e., $\left.C O R\left(C_{P 2-T 2}, R S 3, L T 3\right), C O R\left(C_{P 2-T 2}, L T 3, R P 3\right)\right)$. In general, the rules to infer which instances are correlated are domain and context dependent, and cannot be specified once and for all in a temporal knowledge server. Modelling such rules is outside the goals of this 
paper, where we suppose to have correlations in input, and use them in order to perform temporal reasoning (further discussions on correlation are in [Morris et al., 93; Terenziani, 97; Terenziani, 00]).

Temporal Reasoning. In this paper, as well as in most AI temporal reasoning approaches, we are interested in checking the consistency of temporal constraints ${ }^{2}$. Temporal consistency can be checked on the classes alone, on the instances alone, or on the merge of the constraints on classes and the constraints on instances (i.e., considering inheritance). However, many researchers noticed that "data explicitly stored in a temporal database are often associated with certain semantics assumptions" [Bettini et al., 98, page 277] (the same observation trivially extends to knowledge bases). Thus, the reasoning steps to be performed in order to check temporal consistency may vary depending on the temporal assumptions that underlie the semantics of temporal constraints. In particular, at least two orthogonal assumptions underlie the implicit meaning of temporal constraints over classes/instances of events: (i) the assumption that all instances of events started before now (i.e., that no instance is in the future) and (ii) the assumption that all instances that occur in the given domain are actually observed (i.e., there is full and complete observability of instances of events).

Events in the future. The knowledge base could also contain instances that will occur in the future. This is usual in many temporal Database approaches, where "pro-active" updates are taken into account (e.g., the DB may contain the future event instance that John will have a 5K salary starting from next October). The impact of this assumption on the temporal reasoner is the following: if an instance $i$ is asserted (i.e., inserted in the Knowledge Base), but no information is given on its temporal location, the only temporal assumption is that $i$ starts and ends between $-\infty$ and $+\infty$. On the other hand, if no instances in the future are allowed, whenever an instance is asserted, its starting point is equal or before now.

Complete observability of instances. A plan (or protocol, or guideline) is "predictive", in the sense that, if one has observed a given action E1 which is an instance of a class of events E in a plan, and the class E' follows $\mathrm{E}$ in the general plan, one expects to observe an instance of $\mathrm{E}^{\prime}$ in a time consistent with the temporal constraints between the classes of events E and E' in the plan. In domains where one is certain to have a full and complete observability of instances of events, the consistency check of the temporal constraints must take into account "prediction", since not having observed a given instance of event in a given range of time may indicate an

\footnotetext{
${ }^{2}$ In the temporal case some approaches are also interested in finding realizations of consistent sets of temporal constraints, but this problem is usually studied independently of consistency checking (see, e.g., [Gerevini \& Cristani, 97; VanBeek, 92])
} 
inconsistency. On the other hand, if observations may be incomplete, the fact that an expected instance has not been observed within its expected time range does not imply an inconsistency, since the predicted instance could have occurred, and not have been observed. In such a case, prediction need not to be considered to check consistency.

Orthogonality. The two above assumptions are (in principle) orthogonal. Depending on the specific application, the knowledge base KB containing the (description of) the instances of events may be such that:

(1) KB contains instances of events in the future and the observation of events is incomplete. In many applications, the knowledge base consists of instances of events observed in the past (but not all instances that occurred have been observed) plus possibly some instance of event that will certainly take place in the future; e.g., future promotions may be included in KB;

(2) KB contains no instance of events in the future and the observation of events is incomplete. This is probably the most common case, since it involves all applications where KB only contains the observed instances of events and not all the instances that actually occurred have been observed; e.g., in general medical applications patient records are not complete; obviously, they only contain data observed until now;

(3) $\mathrm{KB}$ contains instances of events in the future and the observation of events is complete. This case corresponds to "omniscience" in the given domain, i.e., to "deterministic" applications in which all future instances of events can be captured; however, specific applications that only concern a specific frame of time - e.g., the current year; consider, e.g., annual salaries of employees, annual scheduling of courses in a school - may fit these assumptions;

(4) KB contains no instance of events in the future and the observation of events is complete. This case may occur, e.g., in monitoring tasks, in which the knowledge base contains all the instances of events observed until now; e.g., in medical monitoring applications patient records may be assumed to be complete; obviously, they only contain data observed until now.

Classes+instances Temporal Manager. To summarize, the goal of the work described in this paper is to propose a general purpose knowledge server (temporal manager) which offers a support for:

- explicitly representing (i) temporal constraints between classes and instances of events, (ii) instance-of relations, and (iii) correlations;

- reasoning about inheritance of temporal constraints, and performing consistency checking in all the four different cases mentioned above. 
On the other hand, in this paper we do not deal with the representation of the internal description of events (which are considered as "primitive” entities; see also the discussions on future works in the concluding section).

A two-layer approach. Before starting to describe the technical aspects of our work, we think it is worth mentioning the main strategy we chose to follow in our approach. We believe that the interaction between temporal constraints between classes of events and temporal constraints between the instances of these events is a complex and interesting problem per se. Therefore, in our current work, we chose to rely on and exploit a wellknown "standard" temporal reasoning approach whenever possible, and to focus on the integration issues. In particular, we chose to adopt the STP framework [Dechter et al., 91], which has been used as the basis for many approaches to temporal reasoning (see, e.g., [Brusoni et al., 97; Meiri, 91] and the survey [Vila, 94]). Unfortunately, the gap between what can be done in STP and what we want to obtain is quite broad. Thus, we chose to adopt a two-layer approach. The lower level is STP, and the upper layer is a high-level language we designed in order to deal with temporal constraints on classes and on instances. Of course, much of our work was done in order to "fill the gap" between the two layers, since "mapping" from the high-level language to the STP "assembly language” means devising suitable algorithms coping with all the above issues (correlation, inheritance, prediction, and so on) $)^{3}$.

\section{$3 \quad$ An integrated approach to temporal reasoning about classes and instances of events}

In the first subsection, we briefly discuss Simple Temporal Problems (henceforth STP [Dechter et al., 91]), a well-consolidated framework which is at the basis of the definition of our temporal language. Then, we describe the languages we propose in order to represent temporal constraints between instances (section 3.2) and classes (section 3.3).

\subsection{STP}

The STP framework is based on the notion of bound on difference constraint. A bound on difference is a linear inequality of the form $d_{1} \leq X-Y \leq d_{2}$ (also strict inequalities are allowed). The variables in a bound on difference may correspond to time points (and extremes of time intervals). Thus, a bound on difference $d_{1} \leq X-Y \leq d_{2}$ may

\footnotetext{
${ }^{3}$ In a certain sense, drawing an intuitive parallel with programming languages, the goal of our work is that of providing users with a new high-level language. In designing such a language, we chose to rely on a "standard" assembly language, and we focused on the design of a suitable compiler (or - better - interpreter) of the highlevel language.
} 
have the following temporal interpretation: the temporal distance between the time points (endpoints of time intervals in our case) $X$ and $Y$ is between $d_{1}$ and $d_{2}(d 1, d 2 \in \mathfrak{R})$. A temporal knowledge base is a conjunction of bounds of differences constraints between variables representing the starting/ending points of events (called STP constraints in [Dechter et al., 91] and in the following). In [Brusoni et al., 97; Meiri, 91] it is shown how different types of temporal constraints can be easily mapped to STP constraints (e.g., this is the case for all the temporal constraints in the examples in sections 3.2 and 3.3 below). In particular, all the qualitative constraints of the Continuous Interval Algebra (i.e., the subset of relations of Allen's Interval Algebra which can be mapped onto conjunctions of constraints between points, excluding inequality [Vila, 94]) can be mapped onto STP constraints, as well as precise and imprecise dates, durations and delays ${ }^{4}$. A set of STP constraints on a set K of variables can be represented in terms of a graph called temporal constraint network $\left(\mathrm{TCN}_{\mathrm{K}}\right.$ in the following), whose nodes correspond to the variables in $\mathrm{K}$. In general, given a temporal constraint network $\mathrm{TCN}_{\mathrm{K}}$, two main problems have to be faced: (i) determining the strictest constraints between each pair of variables (nodes) in K, usually called the minimal network [Dechter et al., 91], and (ii) checking whether the constraints in $\mathrm{TCN}_{\mathrm{K}}$ are consistent. Problem (i) can be solved using a standard all-to-all shortest path algorithm such as, e.g., FloydWarshall's one, which operates in a time cubic in the number of variables (nodes in the graph) and is correct and complete for STP constraints. Moreover, it can also be adapted to check consistency $\left(\mathrm{TCN}_{\mathrm{K}}\right.$ is consistent iff its minimal network does not contain negative cycles [Dechter et al., 91]). Consistency may also be checked by specialised algorithms, such as, e.g., DPC [Dechter et al., 91]. Finally, conjunctions of STP constraints support

efficient query answering [Brusoni et al., 95] and incremental insertion of new constraints [Console \& Terenziani, 99].

\subsection{Language for temporal constraints between instances of events (ITL)}

Time points are the basic notion in our approach. A time interval $I$ is a convex set of points between a starting $(\operatorname{Start}(I))$ and an ending $(\operatorname{End}(I))$ point. Different types of temporal constraints can involve instances of events.

\subsubsection{Temporal constraint language for instances}

\footnotetext{
${ }^{4}$ Notice however that arbitrary disjunctions of temporal constraints (as in "LT1 was during LT2 or LT2 lasted at least 1 hour") cannot be specified by conjunctions of STP constraints, as well as some disjunctive relations in Allen's algebra such as "LT1 before or after LT2", or relative durations such as "LT1 lasted twice than LT2".
} 
Although several approaches directly adopt STP constraints to represent temporal constraints between events, we feel that a temporal knowledge server should propose a higher level interface language to users and applications. In particular, in order to make the interaction with users more friendly, we believe that the temporal manager system should provide commonsense temporal notions such as qualitative relations (e.g., precedence), dates and durations. However, it is important to notice that our high-level constraint language over instances has been designed in such a way that all high-level constructs map onto conjunctions of STP constraints.

Dates locate instances of events in time and can be precise (see Ex.1) or imprecise (Ex.2,3).

(Ex.1) RS1 started on 10/1/00 at 10:00 and ended on 10/1/00 at 10:05.

(Ex.2) RS2 started on 10/1/00 between 10:10 and 10:15 and ended on 10/1/00 at 10:20.

(Ex.3) LT1 started on 15/1/00 at 9:00-9:40 and ended on 15/1/00 at 10:00.

In our language for temporal constraints between instances of events (called ITL) dates can be expressed using the high-level predicate date(E,L1,U1,L2,U2), stating that the starting point of E is a time point temporally located between L1 and $\mathrm{U} 1(\mathrm{~L} 1 \leq \mathrm{U} 1)$ and its ending point is a time point between L2 and $\mathrm{U} 2(\mathrm{~L} 2 \leq \mathrm{U} 2)$. Exact dates (as in Ex.1) can be simply modeled using $\mathrm{L} 1=\mathrm{U} 1$ and $\mathrm{L} 2=\mathrm{U} 2$; unknown extremes can be represented as $-\infty$ and $+\infty$. Also durations can be precise or not (see Ex.4).

(Ex.4) LT2 lasted at least 1 hour.

Durations are represented in ITL by the predicate duration(E,L1,U1), stating that L1 and U1 are the minimum and maximum durations of E respectively (L1 $\leq \mathrm{U} 1)$. Delays (Ex.5) represent the temporal distances between pairs of instances.

(Ex.5) RS2 started 4-5 minutes after the end of RS1.

Delays are represented in ITL by the predicate $\operatorname{delay}(\mathbf{P 1}, \mathbf{P 2}, \mathbf{L 1}, \mathbf{U 1})$, stating that L1 and U1 are the minimum and maximum delay between P1 and P2 $(\mathrm{L} 1 \leq \mathrm{U} 1)$, where P1 and P2 are time points (i.e., starting/ending points of events). On the other hand, qualitative temporal constraints do not involve any metric of time, allowing one to deal with the relative position of two instances of events (Ex.6). Currently, ITL considers all and only the qualitative constraints that can be mapped onto conjunctions of STP constraints (this subset proved to be very important in many practical applications [Brusoni et al., 97; Console \& Terenziani, 99; VanBeek, 92]).

(Ex.6) LT1 was before LT2.

Example. The constraints in (Ex.1-Ex.6) can be represented in ITL as shown by (ITL1).

(ITL1) date(RS1,10/1/00 at 10:00,10/1/00 at $10: 00,10 / 1 / 00$ at $10: 05,10 / 1 / 00$ at 10:05), 
date(RS2,10/1/00 at $10: 10,10 / 1 / 00$ at $10: 15,10 / 1 / 00$ at $10: 20,10 / 1 / 00$ at $10: 20)$,

date(LT1,15/1/00 at 9:00,15/1/00 at 9:40,15/1/00 at 10:00,15/1/00 at 10:00),

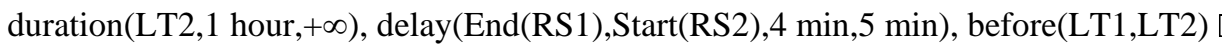

All the constraints in ITL above can be easily mapped onto conjunctions of distances between time points, or, better, into conjunctions of STP constraints.

The semantics of ITL constraints is specified in terms of STP constraints as follows:

date(E,L1,U1,L2,U2) $\Leftrightarrow\left(\mathrm{L} 1 \leq \operatorname{Start}(\mathrm{E})-\mathrm{X}_{0} \leq \mathrm{U} 1\right) \wedge\left(\mathrm{L} 2 \leq \mathrm{End}(\mathrm{E})-\mathrm{X}_{0} \leq \mathrm{U} 2\right)$

duration(E,L1,U1) $\Leftrightarrow \mathrm{L} 1 \leq \operatorname{End}(\mathrm{E})-\operatorname{Start}(\mathrm{E}) \leq \mathrm{U} 1$

$\operatorname{delay}(\mathbf{P 1}$,P2,L1,U1) $\Leftrightarrow \mathrm{L} 1 \leq \mathrm{P} 2-\mathrm{P} 1 \leq \mathrm{U} 1$

Notice that dates are represented by distances from a reference time point $\mathrm{X}_{0}$ for the whole knowledge base. As examples of qualitative relations, let us consider before and during between two time intervals E1 and E2:

before(E1,E2) $\Leftrightarrow 0<\operatorname{Start}(\mathrm{E} 2)-\operatorname{End}(\mathrm{E} 1)$

during(E1,E2) $\Leftrightarrow(0<\operatorname{Start}(E 1)-\operatorname{Start}(E 2)) \wedge(0<\operatorname{End}(E 2)-\operatorname{End}(E 1))$

Additionally, also the constraint that the starting point of an interval precedes the ending point of that interval must be explicitly represented.

Example. Using the (semantic) definitions above, the temporal constraints in (ITL1) can be easily translated into the corresponding set (ITL1') of STP constraints (in ITL1' and in the following examples, we represent by S I $_{\text {I }}$ and $\mathrm{E}_{\mathrm{I}}$ the STP variables corresponding to the starting point Start(I) and the ending point End(I) of a time interval I, and we assume that the reference time $\mathrm{X}_{0}$ is $10 / 1 / 00$ at $0: 00$, and that the granularity is 1 minute).

(ITL1')

$$
\begin{aligned}
& 600 \leq \mathrm{S}_{\mathrm{RS} 1}-\mathrm{X}_{0} \leq 600 \wedge 605 \leq \mathrm{E}_{\mathrm{RS} 1}-\mathrm{X}_{0} \leq 605 \wedge 0 \leq \mathrm{E}_{\mathrm{RS} 1}-\mathrm{S}_{\mathrm{RS} 1} \wedge \\
& 610 \leq \mathrm{S}_{\mathrm{RS} 2}-\mathrm{X}_{0} \leq 615 \wedge 620 \leq \mathrm{E}_{\mathrm{RS} 2}-\mathrm{X}_{0} \leq 620 \wedge 0 \leq \mathrm{E}_{\mathrm{RS} 2}-\mathrm{S}_{\mathrm{RS} 2} \wedge \\
& 6300 \leq \mathrm{S}_{\mathrm{LT} 1}-\mathrm{X}_{0} \leq 6340 \wedge 6360 \leq \mathrm{E}_{\mathrm{LT} 1}-\mathrm{X}_{0} \leq 6360 \wedge 0 \leq \mathrm{E}_{\mathrm{LT} 1}-\mathrm{S}_{\mathrm{LT} 1} \wedge \\
& 60 \leq \mathrm{E}_{\mathrm{LT} 2}-\mathrm{S}_{\mathrm{LT} 2} \wedge 4 \leq \mathrm{S}_{\mathrm{RS} 2}-\mathrm{E}_{\mathrm{RS} 1} \leq 5 \wedge 0<\mathrm{S}_{\mathrm{LT} 2}-\mathrm{E}_{\mathrm{LT} 1}
\end{aligned}
$$

Temporal reasoning (via Floyd-Warshall's all-to-all shortest path algorithm) on (ITL1') finds the minimal and maximal distance between each pair of points (e.g., it infers that RS2 started at 10:10 and LT2 started after 15/1/00 at 10:00), as shown in table 1 . 


\begin{tabular}{l|l|l|l|l|l|l|l|l|l} 
& $\mathbf{X}_{\mathbf{0}}$ & $\mathbf{S}_{\mathbf{R S 1}}$ & $\mathbf{E}_{\mathbf{R} 1}$ & $\mathbf{S}_{\mathbf{L T 1}}$ & $\mathbf{E}_{\mathbf{L T 1}}$ & $\mathbf{S}_{\mathbf{R S} 2}$ & $\mathbf{E}_{\mathbf{R S 2}}$ & $\mathbf{S}_{\mathbf{L T} 2}$ & $\mathbf{E}_{\mathbf{L T 2}}$ \\
\hline $\mathbf{X}_{\mathbf{0}}$ & 0 & 600 & 605 & 6340 & 6360 & 610 & 620 & inf & inf \\
\hline $\mathbf{S}_{\mathbf{R S 1}}$ & -600 & 0 & 5 & 5740 & 5760 & 10 & 20 & inf & inf \\
\hline $\mathbf{E}_{\mathbf{R S 1}}$ & -605 & -5 & 0 & 5735 & 5755 & 5 & 15 & inf & inf \\
\hline $\mathbf{S}_{\mathbf{L T 1}}$ & -6300 & -5700 & -5695 & 0 & 60 & -5690 & -5680 & inf & inf \\
\hline $\mathbf{E}_{\mathbf{L T 1}}$ & -6360 & -5760 & -5755 & -20 & 0 & -5750 & -5740 & inf & inf \\
\hline $\mathbf{S}_{\mathbf{R S} 2}$ & -610 & -10 & -5 & 5730 & 5750 & 0 & 10 & inf & inf \\
\hline $\mathbf{E}_{\mathbf{R S} 2}$ & -620 & -20 & -15 & 5720 & 5740 & -10 & 0 & inf & inf \\
\hline $\mathbf{S}_{\mathbf{L T 2}}$ & -6360 & -5760 & -5755 & -20 & 0 & -5750 & -5740 & 0 & inf \\
\hline $\mathbf{E}_{\mathbf{L T 2}}$ & -6420 & -5820 & -5815 & -80 & -60 & -5810 & -5800 & -60 & 0
\end{tabular}

Table 1. Minimal network for the (consistent) set of constraints (ITL1'). "inf” stands for "infinite”. Notice that, in the table, $T[\mathrm{i}, \mathrm{j}]=\mathrm{b}$ and $\mathrm{T}[\mathrm{j}, \mathrm{i}]=-\mathrm{a}$ conjunctively represent the bound on difference $\mathrm{a} \leq \mathrm{j}-\mathrm{i} \leq \mathrm{b}$.

\subsubsection{Other constructs of ITL}

The temporal constraint language above is very similar to the ones of many temporal managers in the AI literature (see, e.g., [Brusoni et al., 97; Dechter et al., 91; Meiri, 91; Vila, 94]). In order to be able to integrate temporal constraints between classes and between instances, we must extend ITL. We introduce the primitive Instance_of(E1,C1) to state that E1 is a specific instance of the class of events C1.

Example. In the following, we suppose to have the classes in Figure 1, and to have observed only the instances RS1 and RS2 (of Reservation) and LT1, LT2 (of Lab_Tests). The class/instance relations can be represented in ITL as shown by (ITL2):

$$
\begin{array}{lll}
\text { (ITL2) } & \text { Instance_of(RS1, Reservation), } & \text { Instance_of(RS2, Reservation), } \\
& \text { Instance_of(LT1, Lab_Tests), } & \text { Instance_of(LT2, Lab_Tests) }
\end{array}
$$

The primitive COR is introduced to represent correlations between instances of events.

Example. In our example, we assume (as in Figure 1) that LT1 is correlated to RS1 (via the correlation relation $\mathrm{C}_{1}$ ) and LT2 is correlated to RS2 (via the correlation relation $\mathrm{C}_{2}$ ). This can be expressed in ITL by (ITL3):

$$
\text { (ITL3) } \operatorname{COR}\left(\mathrm{C}_{1}, \mathrm{RS} 1, \mathrm{LT} 1\right), \mathrm{COR}\left(\mathrm{C}_{2}, \mathrm{RS} 2, \mathrm{LT} 2\right) \square
$$

Finally, it is useful to indicate explicitly the set IKB_Elements of all the instances e.g., as shown in (ITL4):

(ITL4) \{RS1,RS2,LT1,LT2 $\}.$ 


\subsubsection{Knowledge Base of Instances}

In ITL, a Knowledge Base about instances of events (IKB for short) is a quadruple <IKB_Elements, IKB_Instance_of, IKB_COR, IKB_Constraints>, where IKB_Elements is a set of instances of events, IKB_Instance_of is a set of Instance_of assertions, IKB_COR a set of correlations and IKB_Constraints a set of (i.e., a conjunction of) temporal constraints on instances of events. In our example, we have IKB $=<$ ITL4,ITL2,ITL3,ITL1 $>$.

While the semantics of temporal constraints in our language has been simply expressed in terms of STP constraints, the implicit temporal meaning of having a given instance $i$ in the IKB_Elements component of IKB depends on the assumptions one makes regarding the possibility of dealing with future instances of events in IKB and on whether IKB is complete or not. In the following, we introduce such issues mainly through first order formulae, which thus can be seen as a declarative specification of some of the inferences that the classes+instances temporal reasoning algorithms have to perform ${ }^{5}$.

(1) (Ax1) covers the cases in which the IKB is a representation of the instances of events which have been observed until NOW (where NOW is the system time when a call to the temporal manager is done; i.e., future instances of events cannot be inserted in IKB_Elements):

$$
\forall \mathrm{x} x \in \mathrm{IKB} \_ \text {Elements } \Rightarrow \operatorname{Start}(\mathrm{x})-\mathrm{NOW} \leq 0
$$

(Ax1) states that if an instance $\mathrm{x}$ of event has been observed (i.e., $\mathrm{x} \in \mathrm{IKB}$ _Elements), then it has been observed to start before (or at the same time than) NOW (otherwise, if also future instances are taken into account in IKB, the fact that $\mathrm{x} \in \mathrm{IKB} \_$Elements does not provide any implicit indication on the temporal location of $\mathrm{x}$ ).

(2) In case we assume that observations are complete, the fact that an instance $x$ of event has not been observed (i.e., $\mathrm{x} \notin \mathrm{IKB}$ _Elements) has different temporal implications, depending on whether the system takes into account future events or not. With complete observations and no future events, each instance that does not belong to IKB_Elements has not occurred yet (i.e., did not start before now; this can be formalized as in Ax2).

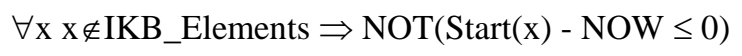

On the other hand, with complete observations and future events, the fact that an event instance does not belong to IKB_Elements implies that it never occurs in the past, present and future.

\footnotetext{
${ }^{5}$ As in Allen’s seminal approach [Allen, 83; Allen, 84; Allen \& Hayes, 85], we use logical formulae to specify formally the intended meaning of our constraint language, and we define specialised temporal reasoning techniques to operate on temporal constraints in an efficient way, respecting the logical semantics of the constraints. In fact, specialised reasoners are usually more efficient than a general purpose theorem prover for a logic [Allen, 83; Allen, 84; Allen \& Hayes, 85].
} 


\subsection{Language for temporal constraints between classes of events (CTL)}

\subsubsection{Temporal constraint language for classes}

In general, all the types of temporal constraints discussed above can also be expressed between classes of events. For instance, considering again the example in Figure 1, one could assert the following temporal constraints:

\section{Example.}

(Ex.7) Laboratory tests are started between 1 and 7 days after the reservation.

(Ex.8) Laboratory tests last between 30 minutes and 48 hours.

(Ex.9) Results are reported between 1 and 48 hours after the end of the tests.

(Ex.10) Results are reported after the tests.

Thus, we used the same constraint language as above to express them into our temporal language for classes of events (CTL for short). Ex.7-Ex.10 are represented in CTL as follows:

(CTL1) Cdelay(End(Reservation),Start(Lab_Tests), 1 day, 7 day), Cduration(Lab_Tests, 30 min, 48 hour), Cdelay(End(Lab_Tests),Start(Report), 1 hour, 48 hour), Cafter(Report,Lab_Tests) $\square$

The temporal constraints on classes are basically the same as for instances (we put the prefix $\mathrm{C}$ to distinguish them). However, the semantics of temporal constraints on classes is different. In fact, when applied to classes, dates, durations, delays (here we consider just the delays between the starting points of two classes; the other cases are analogous) and qualitative relations have a different meaning. This meaning can be expressed, for instance, as shown below. ${ }^{6}$

Cdate(C,L1,U1,L2,U2) $\Leftrightarrow \forall C^{\prime}\left(\right.$ Instance_of(C',C) $\Rightarrow\left(\left(\mathrm{L} 1 \leq \operatorname{Start}\left(\mathrm{C}^{\prime}\right)-\mathrm{X}_{0} \leq \mathrm{U} 1\right) \wedge\left(\mathrm{L} 2 \leq \operatorname{End}\left(\mathrm{C}^{\prime}\right)-\mathrm{X}_{0} \leq \mathrm{U} 2\right)\right)$

Cduration(C,L1,U1) $\Leftrightarrow \forall C^{\prime}$ (Instance_of(C',C) $\Rightarrow$ L1 $\left.\leq \operatorname{End}\left(C^{\prime}\right)-\operatorname{Start}\left(C^{\prime}\right) \leq \mathrm{U} 1\right)$

Cdelay(Start(C1),Start(C2),L1,U1) $\Leftrightarrow(\forall$ C1',C2',Cor (Instance_of(C1',C1) ^ Instance_of(C2',C2) ^ $\left.\left.\mathrm{COR}\left(\mathrm{Cor}, \mathrm{C} 1^{\prime}, \mathrm{C} 2{ }^{\prime}\right)\right) \Rightarrow\left(\mathrm{L} 1 \leq \operatorname{Start}\left(\mathrm{C} 2{ }^{\prime}\right)-\operatorname{Start}\left(\mathrm{C} 1^{\prime}\right) \leq \mathrm{U} 1\right)\right) \wedge$

$(\forall$ C1' Instance_of(C1',C1) $\Rightarrow(\exists$ C2',Cor Instance_of(C2',C2) $\wedge$ COR(Cor,C1',C2'))) $)$

As example of qualitative relations, let us consider the relation "before":

Cbefore(C1,C2) $\Leftrightarrow(\forall$ C1',C2',Cor (Instance_of(C1',C1) $\wedge$ Instance_of(C2',C2) $\wedge$ COR(Cor,C1',C2') $\Rightarrow$ $\left(0<\operatorname{Start}\left(C 22^{\prime}\right)-\operatorname{End}\left(C 1^{\prime}\right)\right) \wedge(\forall$ C1' Instance_of(C1',C1) $\Rightarrow(\exists$ C2',Cor Instance_of(C2',C2) COR(Cor, C1',C2'))))

\footnotetext{
${ }^{6}$ Notice that, as in many AI approaches in the literature (see, e.g., [Nebel, 90]), we specify the semantics of classes in an extensional way, i.e., considering classes as set of instances.
} 
While durations are simply inherited by all instances, qualitative relations and delays are only inherited by correlated pairs of instances (see section 2). The second conjuncts in the definition of Cdelay and Cbefore formalize the "predictive" character of delays and qualitative relations between classes of events. For example, given the constraint between classes Cbefore(C1,C2), the observation of an instance of C1 implies the subsequent occurrence of a correlated instance of C2.

\subsubsection{Other constructs of CTL and Knowledge Base of Classes}

The primitive EventClass is introduced in CTL in order to declare the classes of events being considered. Thus, in the example in Figure 1, we would have (CTL2) below:

(CTL2) EventClass(Reservation), EventClass(Lab_Tests), EventClass(Report).

Thus, in our language CTL, a KB of temporal constraints between classes of events (CKB for short) can be defined as a pair <CKB_EventClass, CKB_Constraints $>(<\text { CTL2,CTL1 }>\text { in our example })^{7}$.

\section{$4 \quad$ Classes+instances consistency checking with incomplete observations and future events}

Usually, temporal constraint languages have been applied to describe temporal constraints on instances of events only [Vila, 94]. Obviously, whenever one considers instances only, there is no need of classes+instances temporal reasoning, and a standard approach such as the one in section 3.1 and 3.2.1 is sufficient. Also in the cases where one has only constraints between classes, the fact that they are classes is irrelevant from the point of view of temporal reasoning; they can be interpreted as primitive (individual) events and standard temporal reasoning can be performed on them (see, e.g., [Allen, 91b] as regards temporal constraints in general plans).

On the other hand, classes+instances temporal reasoning takes in input both a KB of temporal constraints between classes and a KB of temporal constraints between instances, and gives as output the upper and lower bounds on the distance between each pair of starting and ending points of instances (i.e., the minimal network) considering both the instance constraints and the constraints inherited from classes or an inconsistency. The procedure Integrated_Reasoning_IF (“I” stands for Incomplete observations, "F” stands for Future instances of events) in

\footnotetext{
${ }^{7}$ Notice that, in this representation, we assume that the temporal constraints in CKB_Constraints implicitly represent the possible presence of a correlation relation between the corresponding instances. In other words, given two instances E1 and E2 of two concepts $\mathrm{C} 1$ and $\mathrm{C} 2$ respectively, E1 and E2 may be correlated only in case C1 and C2 are related via some temporal relation in the CKB (notice that the relation C1 ALL C2 can be used in order to relate C1 and C2 stating that any temporal constraint can hold between them, i.e., that $-\infty \leq \mathrm{S}_{\mathrm{C} 1}-\mathrm{S}_{\mathrm{C} 2} \leq+\infty \wedge-\infty \leq \mathrm{S}_{\mathrm{C} 1}-\mathrm{E}_{\mathrm{C} 2} \leq+\infty \wedge-\infty \leq \mathrm{E}_{\mathrm{C} 1}-\mathrm{S}_{\mathrm{C} 2} \leq+\infty \wedge-\infty \leq \mathrm{E}_{\mathrm{C} 1}-$ $\left.\mathrm{E}_{\mathrm{C} 2} \leq+\infty\right)$. In other words, we assume (Ax3):

(Ax3) $\forall \mathrm{c} 1 \in \mathrm{CKB} \_$EventClass, $\forall \mathrm{c} 2 \in \mathrm{CKB} \_$EventClass $\quad$ NOT (Connected(CKB,c1,c2) $\Rightarrow(\forall \mathrm{e} 1, \mathrm{e} 2$ Instance_of(e1,c1) $\wedge$ Instance_of(e2,c2) $\Rightarrow$ NOT $\exists$ Cor $\left.\left._{\mathrm{i}}\left(\mathrm{COR}\left(\mathrm{Cor}_{\mathrm{i}}, \mathrm{e} 1, \mathrm{e} 2\right)\right)\right)\right)$

where Connected(c1,c2) informally represents the fact that the classes of events c1 and c2 are connected vertices in the graph formed by the constraints in CKB_Constraints.
} 
Figure 2 considers a CKB and a IKB expressed using the language in section 3 and deals with the case 1 described in section 2, i.e., the case in which observations are incomplete (i.e., instances of events can occur and not be observed) and the IKB may also contain future instances of events. From the algorithmic point of view, this is the easiest case. In section 5, we will then extend Integrated_Reasoning_IF to deal with cases 2, 3 and 4 introduced in section 2.

First of all, Integrated_Reasoning_IF pre-computes all pairs of classes in CKB which are connected via the temporal constraints in CKB_Constraints (in our approach, only the instances of connected classes can be correlated; see footnote 7). This step (step 1) can be easily computed by performing a transitive closure on the graph of constraints. Before performing classes+instances temporal reasoning, temporal constraints in the highlevel language are independently translated into the corresponding set of STP constraints (steps (2) and (3)). This can be easily done on the bases of the definitions given in section 3.2. Then, the propagation of temporal constraints is performed independently on the STP constraints on instances alone (see step 4), and on the STP constraints on classes alone (see step 5) to check whether each one of them is independently consistent and to infer the implied temporal constraints in each of them separately (let STP_IKB_Con' and STP_CKB_Con' the resulting sets of constraints). The propagations of STP temporal constraints at step 4 and 5 can be performed, e.g., using Floyd-Warshall's all-to-all shortest paths algorithm on STP constraints (called here All-Shortest-Paths), as discussed in section 3.1 and exemplified in section 3.2 as regards instances (the case of classes alone is analogous). In case an inconsistency is detected (i.e., in the case the temporal constraints between instances and/or between classes, taken in isolation, are already inconsistent), it is reported and the algorithm stops. Otherwise, symmetric and transitive closure is performed on correlation relations between instances (step 6). For the sake of efficiency, we also suppose that closure pre-computes, for each node, the list of its connected nodes. The rest of the procedure deals with the integration of the two levels of constraints. The basic idea is that of inheriting (accordingly with the semantics specified is section 3.3.1) the temporal constraints between classes on the instances of events, and then performing temporal reasoning on instances (applying again the all-shortest-path algorithm) on the union of the inherited plus the instance constraints. Step (7) simply checks that, for each pair i1,i2 of correlated instances, if C1 and C2 are their corresponding classes, then Connected(CKB,C1,C2) holds (see Ax3 in footnote 7). Step (8) computes the inheritance of temporal constraints, adding the constraints on event classes on the corresponding instances (see below). Finally, step (9) performs integrated reasoning at the level of instances, considering also inherited constraints. The procedure stops reporting an inconsistency if a call to AllShortest-Paths (steps 4, 5, and 9) or the checks in (7) find it. 
Temporal_Constraint_Inheritance operates as follows. Step (1) implements the inheritance of durations and dates of events. For example, as regards durations, all distances $t \leq \mathrm{End}(\mathrm{E})$ - Start $(\mathrm{E}) \leq \mathrm{u}$ between the ending point and the starting point of an event class E must be inherited by all the instances of the class. Thus, they are added to the constraints in STP_IKB_Con'. Step (2) deals with the inheritance of qualitative relations and delays. Step (2) is implemented by (i) looking at correlated pairs <i1,i2> of instances; (ii) considering the corresponding pair $<\mathrm{C} 1$, $\mathrm{C} 2>$ of classes; (iii) considering the STP constraints between $\mathrm{S}_{\mathrm{C} 1}, \mathrm{~S}_{\mathrm{C} 2}, \mathrm{E}_{\mathrm{C} 1}, \mathrm{E}_{\mathrm{C} 2}$, and (iv) asserting them onto $\mathrm{S}_{\mathrm{i} 1}, \mathrm{~S}_{\mathrm{i} 2}, \mathrm{E}_{\mathrm{i} 1}, \mathrm{E}_{\mathrm{i} 2}$.

Procedure Integrated_Reasoning_IF( $<$ CKB_EventClass, CKB_Constraints $>$, $<$ IKB_Elements,IKB_Instance_of, IKB_COR, IKB_Constraints $>$ )

(1) Pre-compute the pairs of connected nodes in $\mathrm{CKB}$;

(2) STP_IKB_Con := Trasform(IKB_Constraints);

(3) STP_CKB_Con := Trasform(CKB_Constraints);

(4) STP_IKB_Con' := All-Shortest-Paths(STP_IKB_Con);

(5) STP_CKB_Con' := All-Shortest-Paths(STP_CKB_Con);

(6) IKB_COR := Closure(IKB_COR);

(7) Check consistency of correlations;

(8) Temporal_Constraint_Inheritance(CKB,STP_CKB_Con',IKB,STP_IKB_Con');

(9) Minimal_Network := All-Shortest-Paths(STP_IKB_Con');

Procedure Temporal_Constraint_Inheritance(CKB,STP_CKB_Con',IKB,STP_IKB_Con')

(1) Forall $E \in$ IKB_Elements do

Let $\mathrm{C} \in \mathrm{CKB}$ _EventClass the corresponding class

/* i.e., Instance_of(E,C) holds */

Let $\mathrm{t} \leq \operatorname{End}(\mathrm{C})-\operatorname{Start}(\mathrm{C}) \leq \mathrm{u} \in \mathrm{STP} \_\mathrm{CKB} \_$Con' the constraint on the duration of $\mathrm{C}$ (if any)

STP_IKB_Con' := STP_IKB_Con' $\cup\{\mathrm{t} \leq \operatorname{End}(\mathrm{E})-\operatorname{Start}(\mathrm{E}) \leq \mathrm{u}\}$;

Let $\mathrm{t} 1 \leq \operatorname{Start}(\mathrm{C})-\mathrm{X}_{0} \leq \mathrm{u} 1 \in \mathrm{STP} \_$CKB_Con' and $\mathrm{t} 2 \leq \operatorname{End}(\mathrm{C})-\mathrm{X}_{0} \leq \mathrm{u} 2 \in \mathrm{STP} \_$CKB_Con' the constraints on the date of $\mathrm{C}$ (if any)

STP_IKB_Con' := STP_IKB_Con' $\cup\left\{\mathrm{t} 1 \leq \operatorname{Start}(\mathrm{E})-\mathrm{X}_{0} \leq \mathrm{u} 1, \mathrm{t} 2 \leq \operatorname{End}(\mathrm{E})-\mathrm{X}_{0} \leq \mathrm{u} 2\right\}$; od;

(2) Forall E1,E2 $\in$ IKB_Elements, E1 $\neq$ E2 $\backslash$ Exists Cor COR(Cor,E1,E2) do

Let $\mathrm{C} 1 \in \mathrm{CKB} \_$EventClass and C2 $\in \mathrm{CKB}$ _EventClass the corresponding classes

/* i.e., Instance_of(E1,C1) and Instance_of(E2,C2) hold */

Instantiate on E1 and E2 the constraints in CKB_Constraints between C1 and C2; od;

Figure 2. Procedure Integrated_Reasoning_IF.

Complexity ${ }^{8}$. Integrated_Reasoning_IF operates in a time $\mathrm{O}\left(\operatorname{Max}\left(\mathrm{N}^{3}, \mathrm{M}^{3}, \mathrm{~N}^{*} \mathrm{M}^{2}\right)\right)$, where $N$ is the number of classes in $C K B$ and $M$ is the number of instances in IKB. In fact, steps (1) and (6) perform transitive closures (cubic time on the number of nodes), and build, for each node, the list of connected (for classes) or correlated (for

${ }^{8}$ In the analysis of the computational complexity of this algorithm and of the following ones, we assume a suitable implementation of the data contained in CKB and IKB. In particular, we assume that (i) each class is associated with a pointer to the list of its instances, and with a pointer to the list of the other classes connected with it via an input temporal constraint (i.e., to correlated classes of events), (ii) each instance is associated with a pointer to its class, and with a pointer to each list of the other instances that are correlated to it via a correlation relation, (iii) that the access to each instance or class can be achieved in constant time. 
instances) nodes. Steps (4), (5), and (9) apply Floyd-Warshall's algorithm (cubic time on the number of nodes), steps 2 and 3 can be performed in linear time on the number of constraints [Console \& Terenziani, 99]. Given the pre-computation in step (1), and since, for each class in CKB, there are at most $\mathrm{N}$ connected classes, step (7) is performed in time $\mathrm{O}\left(\mathrm{N}^{*} \mathrm{M}^{2}\right)$, where $\mathrm{M}^{2}$ is (in the worst case) the number of correlated pairs of instances. As regards temporal constraints inheritance, step (1) requires linear time on the number of instances (which are at most M); step (2) computes inheritance of qualitative constraints and delays. For each correlated pair <i1,i2> of instances (at most: $\mathrm{O}\left(\mathrm{M}^{2}\right)$ ) it inherits the constraints (if any) from their classes (i.e., it takes the constraints on the four endpoints of the classes and asserts them in IKB on the corresponding endpoints of i1 and i2) 9

Example. For example, let us apply Integrated_Reasoning_IF to <CTL2,CTL1> and <ITL4,ITL2,ITL3,ITL1> described above, supposing that NOW=18/1/00 at 18:00. The translation of ITL1 into STP constraints is ITL1' (see section 3.2.1), while the translation of CTL1 is the set CTL1' (where explicit bounds on differences have been added in order to model the fact that the starting point of an interval precedes its ending point):

(CTL1')

$$
\begin{aligned}
& 1440 \leq \mathrm{S}_{\mathrm{LT}}-\mathrm{E}_{\mathrm{RS}} \leq 10080 \wedge 30 \leq \mathrm{E}_{\mathrm{LT}}-\mathrm{S}_{\mathrm{LT}} \leq 2880 \wedge \\
& 60 \leq \mathrm{S}_{\mathrm{RP}}-\mathrm{E}_{\mathrm{LT}} \leq 2880 \wedge 0<\mathrm{S}_{\mathrm{RP}}-\mathrm{E}_{\mathrm{LT}} \wedge \\
& 0<\mathrm{E}_{\mathrm{RS}}-\mathrm{S}_{\mathrm{RS}} \wedge 0<\mathrm{E}_{\mathrm{LT}}-\mathrm{S}_{\mathrm{LT}} \wedge 0<\mathrm{E}_{\mathrm{RP}}-\mathrm{S}_{\mathrm{RP}}
\end{aligned}
$$

The application of All-Shortest-Paths to ITL1' does not discover any inconsistency, and gives as result the minimal network in Table 1 (see Section 3.2.1). Analogously, application of All-Shortest-Paths to CTL1' does not discover any inconsistency, and gives as result the minimal network in Table 2 below.

\begin{tabular}{|l|l|l|l|l|l|l|}
\hline & SRS & ERS & SLT & ELT & SRP & ERP \\
\hline SRS & 0 & inf & inf & inf & inf & inf \\
\hline ERS & 0 & 0 & 10080 & 12960 & 15780 & 15720 \\
\hline SLT & -1440 & -1440 & 0 & 2880 & 5700 & 5640 \\
\hline ELt & -1470 & -1470 & -30 & 0 & 2820 & 2760 \\
\hline SRP & -1590 & -1590 & -150 & -120 & -60 & -120 \\
\hline ERP & -1590 & -1590 & -150 & -120 & -60 & -120 \\
\hline
\end{tabular}

Table 2. Minimal network.

\footnotetext{
${ }^{9}$ For the sake of efficiency, in many applications some of the steps of procedure Integrated_Reasoning_IF (e.g. steps 1,3 , and 5) can be pre-computed a priori.
} 
Step (1) of Temporal_Constraint_Inheritance inherits the constraints on the duration of LT1 and LT2 (which must last between 30 minutes and 48 hours). Step (2) of Temporal_Constraint_Inheritance inherits the delay of 1-7 days between correlated pairs of instances of Reservation and Lab_Tests. In the example, and taking minutes as the basic granularity, this corresponds to adding the constraints $30 \leq \mathrm{E}_{\mathrm{LT1} 1}-\mathrm{S}_{\mathrm{LT} 1} \leq 2880, \quad 30 \leq \mathrm{E}_{\mathrm{LT2}}-\mathrm{S}_{\mathrm{LT} 2} \leq 2880$, $1440 \leq \mathrm{S}_{\mathrm{LT} 1}-\mathrm{E}_{\mathrm{RS} 1} \leq 10080,1440 \leq \mathrm{S}_{\mathrm{LT2} 2}-\mathrm{E}_{\mathrm{RS} 2} \leq 10080$ into the temporal constraints between instances of events. Analogously, in step (2) also the delay between Lab_Tests and Reports is inherited. The final application of AllShortest-Paths (step 9) does not detect any inconsistency and provides, among the others, the constraints that:

LT1 starts on 15/1/00 at 9-9:30; LT2 starts between 15/1/00 at 10:00 and 17/1/00 at 10:20 and ends between $15 / 1 / 00$ at $11: 00$ and $19 / 1 / 00$ at $10: 20$.

More generally, the following property holds:

Property 1 The procedure Integrated_Reasoning_IF is correct with respect to the semantics of the temporal language we introduced in subsections 3.2 and 3.3 .

Proof (Sketch) The proof is based on the fact that all and only the temporal constraints specified by the semantics of the constructs in CTL (Cdate, Cduration, Cdelay etc.) are inherited at the level of instances of events (step 8), and then correct and complete temporal reasoning is performed at the level of instances of events via the all-to-all shortest path algorithm (step 9).

Given Property 1 above, Integrated_Reasoning_IF is complete as regards consistency checking on the classes in CKB plus the instances in IKB. Integrated_Reasoning_IF does not consider the "predictive” part in the logical semantics of delays and qualitative relations between classes, since it does not add the predicted (correlated) instances into the IKB. However, since now we are considering the case of incomplete observations (i.e., the formula Ax2 does not hold), prediction has no impact on consistency checking. In fact, even if the predicted events should have occurred in the past (i.e., before NOW), not having them in the IKB does not imply an inconsistency: maybe they occurred and were not observed (inserted in the IKB). Moreover, since future events are considered, the fact that an instance is contained into IKB_Elements does not imply anything on its location in time (e.g., Ax2 does not apply to this case). Thus, Property 2 holds:

Property 2 In the case of incomplete observations and future events, the procedure Integrated_Reasoning_IF checks consistency in a correct and complete way with respect to the semantics of the temporal language.

\section{Extensions of the classes+instances consistency checking algorithm}




\subsection{Incomplete observations with no future events}

The extension to deal with the fact that no future instance of event can be present in IKB_Elements (i.e., to cover case 2 in section 2) is quite trivial. In fact, we only need to augment the algorithm Integrated_Reasoning_IF with an additional step (to be performed between step 3 and step 4) stating that each instance in IKB_Elements should have started before (or equal to) now (i.e., the additional step is the procedural implementation of axiom Ax1 in section 3.2.3). We call Integrated_Reasoning_IP (“P” stands for Past or present) the resulting algorithm.

Example. In our example, we have observed (the beginning of) LT2, but there is only the constraint before(LT1,LT2) concerning LT2 in IKB. Thus, in the mapping on STP constraints, we have $S_{L T 2}-X_{0}<\infty$, and the effect of the additional step is to change this constraint into $S_{L T 2}-X_{0} \leq N O W$.

\subsection{Complete observations with future events}

In applications where one can hypothesize that observations are complete (i.e., the formula Ax2 holds), "prediction" must be used to detect inconsistency. In fact, in such a case, the absence of the observation of an instance of an event which, according to the constraints among classes, should happen (and thus be present in IKB), gives an inconsistency. This can be coped with as in procedure Integrated_Reasoning_CF in Figure 3.

The procedure first calls Integrated_Reasoning_IF (step 1) and then considers “predictions”. In the procedure, we denote by CKB_Connected(C) the set of all classes C' in CKB that can be reached (directly or indirectly) from the class C via some temporal constraint (a delay or a qualitative relation), i.e., such that Connected(CKB,C,C') holds. Step (2) implements the "prediction" of new instances. For each instance E in IKB, it considers all the classes in CKB_Connected $\left(\mathrm{C}_{\mathrm{E}}\right)$ which are connected to the class $\mathrm{C}_{\mathrm{E}}$ of which $\mathrm{E}$ is an instance. For each one of these classes (say C'), it looks whether there is an instance E' of C which is correlated to E in IKB. If there is not, an inconsistency is reported, and the algorithm stops. In fact, the hypothesis of full observability, together with the fact that IKB also contains future events, implies that all instances that occurred, are occurring or will occur are described in IKB. Thus, if there is no E' correlated to E in IKB, this means that such E' does not exist, and this is inconsistent with the "predictive" part of the semantics of delays and qualitative constraints between classes (that predicts the existence of a correlated instance). 
Procedure Integrated_Reasoning_CF $\left(<\mathrm{CKB} \_\right.$EventClass, CKB_Constraints $>$,

$<$ IKB_Elements,IKB_Instance_of, IKB_COR, IKB_Constraints $>$ )

(1) Integrated_Reasoning_IF (CKB,IKB);

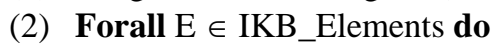

Let $C_{E} \in$ CKB_EventClass the class such that Instance_of(E,CE) $\in$ IKB_Instance_of

Let $\mathrm{CKB}$ _Connected $\left(\mathrm{C}_{\mathrm{E}}\right)$ the set of all classes in $\mathrm{CKB}$ _EventClass connected to $\mathrm{C}_{\mathrm{E}}$ via temporal constraints

Forall $C \in$ CKB_Connected( $\mathrm{C} E)$ do

if NOT (Exists E',Cor' such that Instance_of(E',C) $\in$ IKB_Instance_of $\wedge$ COR(Cor',E',E) $\in$ IKB_COR)

then INCONSISTENT; od; od;

Figure 3. Procedure Integrated_Reasoning_CF.

Complexity. The complexity of Integrated_Reasoning_CF is $\mathrm{O}\left(\max \left(\mathrm{N}^{3}, \mathrm{M}^{3}, \mathrm{~N}^{*} \mathrm{M}^{2}\right)\right)$. In fact, supposing that, for each instance, we pre-compute the lists of its correlated instances (which are, at most, M), the additional step (2) requires at most $\mathrm{O}\left(\mathrm{N}^{*} \mathrm{M}^{2}\right)$ operations.

Example. The CKB “predicts” the existence of two instances of RP, one correlated to LT1 and one correlated to LT2. Since such instances are not present in IKB, step (2) above reports an inconsistency.

\subsection{Complete observations with no future event}

Also in applications where one can hypothesize that observations are complete, but IKB only concerns instances until "NOW" (i.e., the formula Ax2 holds), "prediction" must be used to detect inconsistency. However, in such a case, the check is more complex than in the case we discussed in section 5.2. In fact, let us suppose that the CKB predicts the occurrence of an instance $i$ of event, and that $i$ is not present in IKB. This fact is inconsistent only in case the temporal constraints in IKB and CKB are such that they impose that $i$ must have been observed before (or at the same time than) NOW. Otherwise, no inconsistency arises, since $i$ might be observed (and inserted in the IKB) in the future. This case can be coped with as in Procedure Integrated_Reasoning_CP in Figure 4. NEW_INST is a (initially empty) set containing the "predicted" instances (which are not yet present in IKB). First, the procedure calls Integrated_Reasoning_IP. Then, it checks for inconsistencies with predictions. The idea is very simple. Whenever a predicted instance is detected (and is not yet present in IKB), the procedure "hypothesizes" the occurrence of such an instance, which is provisionally inserted in IKB (and in NEW_INST). Then constraint inheritance is applied to both "real" and "hypothesized" instances, and the propagation of 
temporal constraints in IKB is performed on both types of instances. Finally, Integrated_Reasoning_CP checks whether the resulting temporal constraints are such that a "hypothesized" instance should necessarily have occurred before NOW. Only in such a case, an inconsistency is reported. In step (3.1.1) Add_Instance(C, IKB_Elements, IKB_Instance_of, IKB_COR, E) returns a new instance I’ of C and inserts it into IKB_Elements, augmenting IKB_Instance_of and IKB_COR accordingly; this amounts to creating a new instance I' of C correlated to E, according to the "predictive" part of the semantics of the constraints between classes. Finally, step (7) checks, for each one of the new instances $I$ introduced into the IKB (the instances in NEW_INST), whether the resulting constraints in the IKB imply that $I$ should have started necessarily before NOW (NEC(KB,test) holds if test is necessarily true given the constraints in KB -i.e., if test is logically implied by KB).

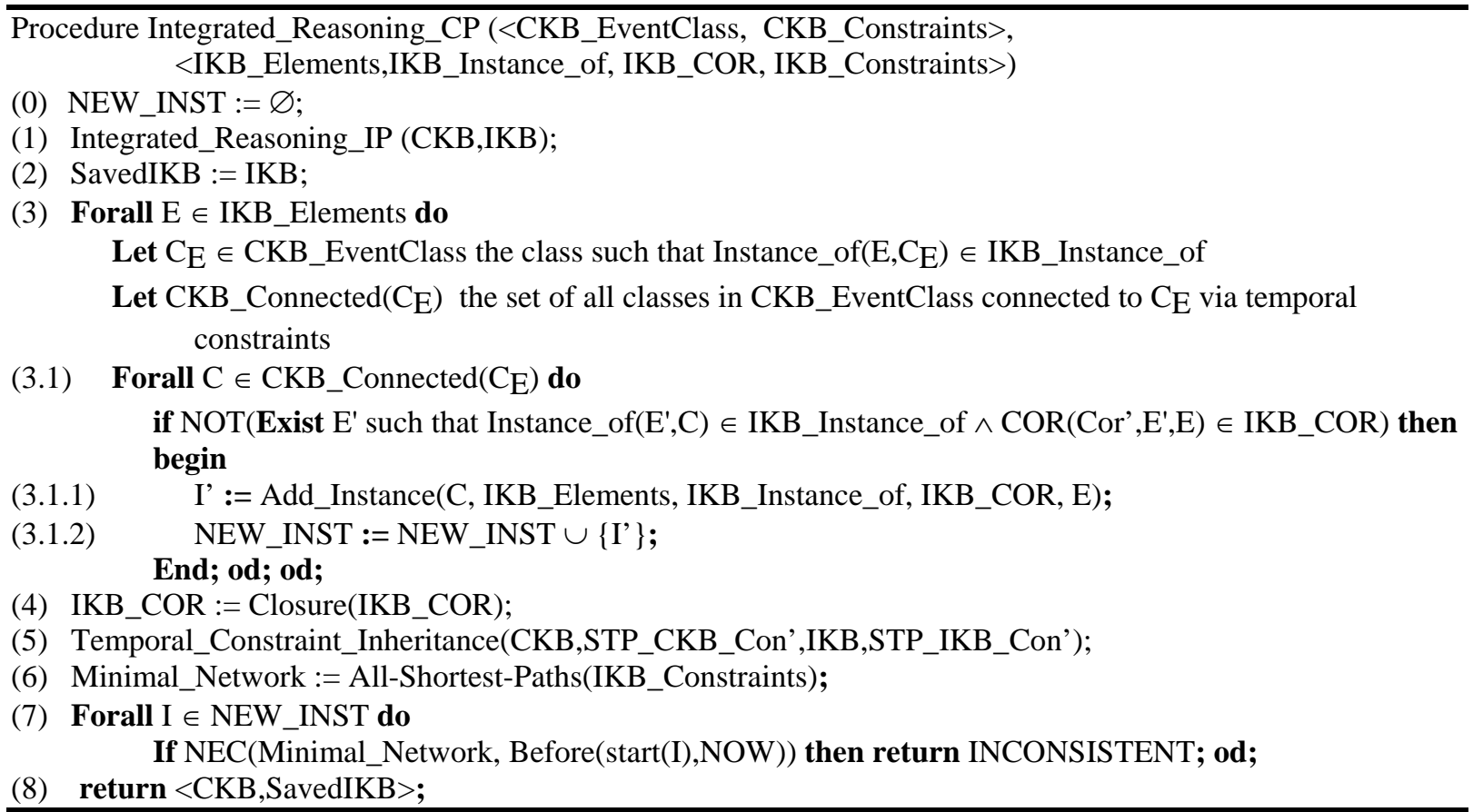

Figure 4. Procedure Integrated_Reasoning_CP.

Complexity. The overall complexity of Integrated_Reasoning_CP also depends on the number $\mathrm{H}$ of "hypothesized” instances (see step 3). The cost of Integrated_Reasoning_IP in step (1) is O(Max $\left(\mathrm{N}^{3}, \mathrm{M}^{3}, \mathrm{~N}^{*} \mathrm{M}^{2}\right)$ ). The cost of step (3), which "hypothesizes" the predicted instances, is $\mathrm{O}\left(\mathrm{N}^{*} \mathrm{M}^{2}\right)$. After step (3), IKB has been modified in order to contain also the new ("hypothesized") instances (say H new instances). Later steps in the algorithm take into account also such instances. Thus, step (4) requires $\mathrm{O}\left((\mathrm{M}+\mathrm{H})^{3}\right)$, step (5) requires $\mathrm{O}\left((\mathrm{M}+\mathrm{H})^{2}\right)$, step (6) $\mathrm{O}\left((\mathrm{M}+\mathrm{H})^{3}\right)$. Finally, let us consider the complexity of step (7). One possibility (as suggested in 
[VanBeek, 91] as regards a language considering qualitative constraints only) is to perform each test NEC(Minimal-Network, Before(Start(I),NOW)) in two steps: (1) provisionally adding the constraint After(Start(I),NOW) to the Minimal Network, and (2) performing temporal reasoning to check consistency. Adopting such a strategy, the cost of $(7)$ would be $\mathrm{O}\left(\mathrm{H}^{*}(\mathrm{M}+\mathrm{H})^{3}\right)$. However, exploiting the locality properties of STP constraints that we proved in [Brusoni et al., 95] (and given the fact that step (6) computes a minimal network), step (7) can be done in a time linear in the time points in NEW_INST, i.e., in time $\mathrm{O}(\mathrm{H})$. Thus, the overall complexity is $\mathrm{O}\left(\operatorname{Max}\left(\mathrm{N}^{3},(\mathrm{M}+\mathrm{H})^{3}, \mathrm{~N}^{*} \mathrm{M}^{2}\right)\right.$. In the worst case (in which the CKB forms a N-length chain of -correlated- classes related by temporal constraints, and that each one of the $\mathrm{M}$ instances corresponds to a different instantiation of such a chain - i.e., no instance in IKB is correlated with any other) we have $\mathrm{H}=\mathrm{N}^{*} \mathrm{M}$.

Example. Let us consider again our example. The procedure above inserts two instances RP1 and RP2 of Report into the IKB. RP1 is correlated to RS1 and LT1, and RP2 to RS2 and LT2. Considering the inherited temporal constraints, we infer that RP1 should start between 1 and 48 hours after the end of LT1, i.e., between 15/1/00 at 11:00 and 17/1/00 at 10:00, and RP2 should start between $15 / 1 / 00$ at 12:00 and 21/1/00 at 10:20. In particular, the starting point of RP1 must be between $15 / 1 / 00$ at $11: 00$ and $17 / 1 / 00$ at 10:00 and thus it is necessarily before NOW (18/1/00 at 18:00 in our example). Thus, an inconsistency is detected.

\subsection{Properties}

Property 3 holds:

Property 3. The procedures Integrated_Reasoning_IP, Integrated_Reasoning_CF, and Integrated_Reasoning_CP check consistency in a correct and complete way with respect to the semantics of the temporal language we introduced in subsections 3.2 and 3.3.

Proof (Sketch) Let us consider, e.g., procedure Integrated_Reasoning_CP (the other cases are easier). The proof is based on the fact that Integrated_Reasoning_IP is correct, and its incompleteness is only due to the fact that it does not consider the "predictive" part of the semantics of constraints between classes, which is dealt with by step (3) of Integrated_Reasoning_CP. Then, the transitive closure of the COR relation is performed to explicit all possible correlations. Correlations are then used in step (5), which performs temporal constraint inheritance in a complete and correct way (accordingly with the constraint semantics discussed in section 3.3.1). Then correct and complete temporal reasoning is performed at the level of instances of events via the all-to-all shortest path 
algorithm (step 6). Finally, step 7 is needed to force the fact that observations are complete (see Ax2), checking whether some predicted instance should have been observed necessarily before now.

\section{An application to clinical guidelines: a preliminary report}

We regard our work as a first step to build a domain and task independent knowledge server system. Although it can operate as a stand-alone system, we believe that, in most cases, it can be loosely coupled with other systems and problem solvers to deal with different problems in different areas, following the lines which have been pointed out, e.g., by [Allen, 91a; Brusoni et al., 97] as regards applications of temporal managers dealing with instances only. Figure 5 shows the general architecture we envision: TKS stands for the Temporal Classes+instances Knowledge Server we propose in this paper, TDPS is a Task (and possibly domain) Dependent Problem Solver. The interface module copes with both the interaction with the outside world (e.g., users, applications) and with the interaction between TKS and TDPS. Whenever a problem about temporal constraints arises in TDPS, it is sent (via the Interface Module) to the TKS, which returns (again, via the Interface Module) the appropriate results.

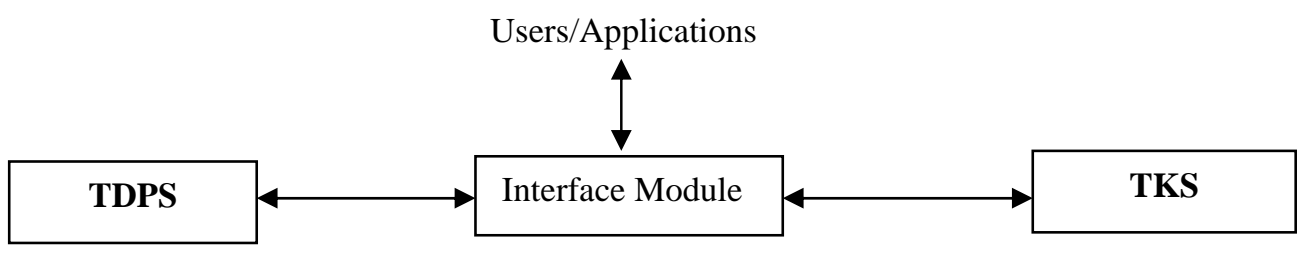

Figure 5. A loosely coupled interaction with the Temporal Knowledge Server. 


\subsection{An application to clinical guidelines}

We have built a first prototype of our knowledge server (in C), and we are starting to apply it to the domain of clinical guidelines management. In the last years, we started a cooperation with physicians of Azienda Ospedaliera S. Giovanni Battista of Torino, Italy, to devise GLARE (GuideLines Acquisition, Representation and Execution system) a computer-based system devoted to the acquisition and the consultation of clinical guidelines [Terenziani et al., 01]. Basically, a clinical guideline is an encoding of the best clinical procedures to diagnose and treat medical diseases. Differently from medical protocols, guidelines are not normative, and propose to physicians different alternatives, giving physicians the freedom of choosing between them. A detailed discussion of the GLARE system is outside the goals of this paper, and can be found in [Terenziani et al., 00; Terenziani et al., 01]. It is just worth mentioning that, currently, GLARE consists of an acquisition module, which acquires clinical guidelines from expert physicians using a graphical interface, and stores it into an internal computer formalism (and, finally, onto a relational DBMS), and a consultation module, which allows physicians to apply general guidelines to specific patients, accessing the patient DB to retrieve patients' data. Figure 6 shows part of the clinical guideline about reflux esophagitis, as it appears to physicians using GLARE's acquisition tool. Different forms of nodes are used in order to represent different types of actions (e.g., "symptom request" represents a data request, "GERD differential diagnosis" represents a decision process and "first line treatment" a composite action). We applied our domain-independent tool to model and consult clinical guidelines on different domains, including bladder cancer, reflux esophagitis, and heart failure.

Temporal constraints play a preminent role in clinical guidelines. In fact, a clinical guideline represents a set of clinical actions (data requests, physical actions, laboratory tests, therapeutic and diagnostic decisions) that are temporally related with each others. Of course, each action in a guideline corresponds to a class of events in our approach, since a guideline may have different instantiations on different patients. Thus, our CTL language can be used during the acquisition phase in order to model the temporal constraints between classes of actions. On the other hand, during the consultation phase, the same or different clinical guidelines may be instantiated on one or more patients. Our ITL language can thus be used to model temporal constraints on instances. In particular, IKB_Elements will contain all the observed instances of actions (i.e., the actions in the guideline that have been already applied to the given patients), and IKB_Instance_of trivially states the guideline action corresponding to each instance. As regards IKB_COR, it is worth noticing that, in this specific domain, correlation is trivially obtained by a rule stating that all and only instances of actions corresponding to the same execution of a given 


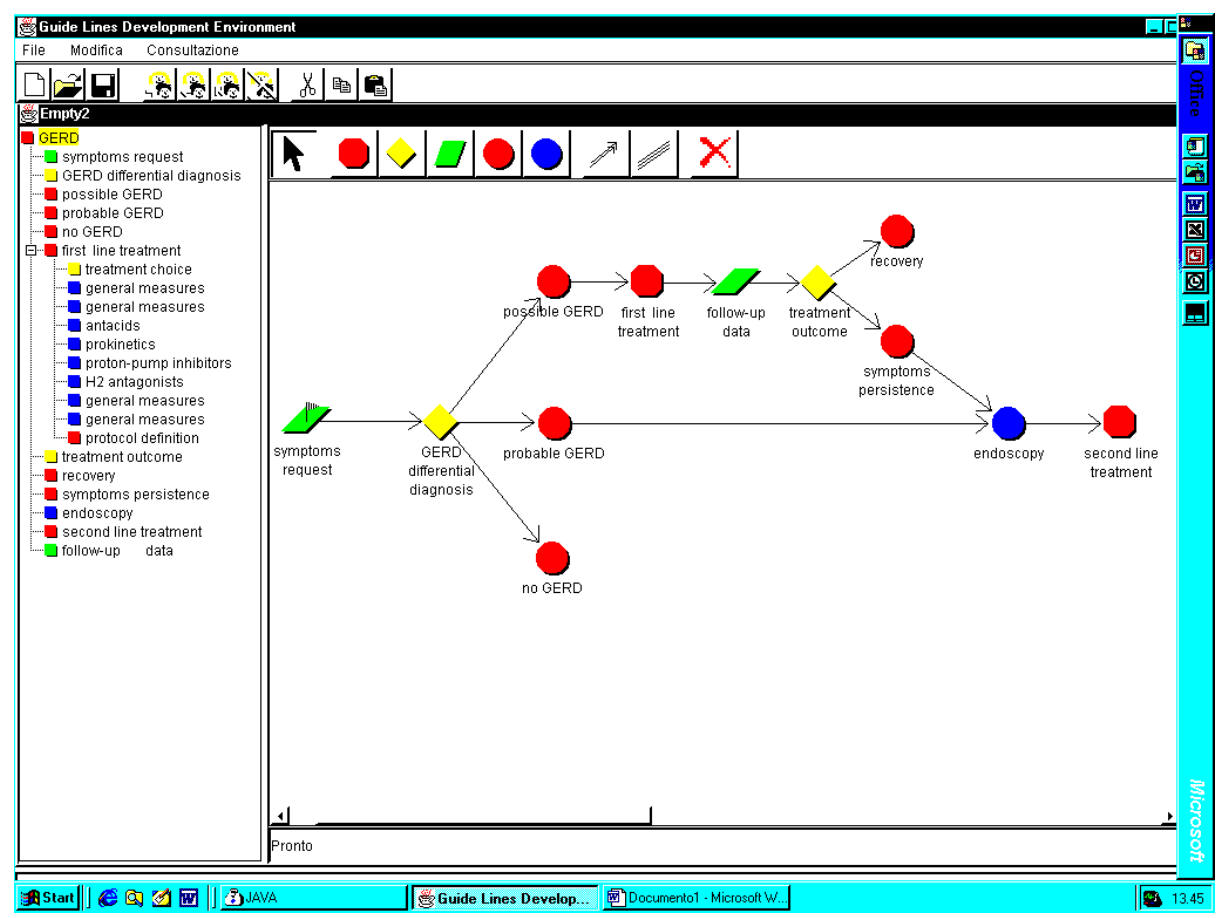

Figure 6. Part of the guideline for reflux esophagitis (GERD for short).

guideline on a given patient are correlated. Finally, IKB_Constraints contains the temporal location of such instances.

In the specific application we are devising, (i) we have only (possibly imprecise) dates as temporal constraints on instances, (ii) we are not allowed to assume full and complete observability, and (iii) there are no events in the future. A preliminary evaluation of such an application is reported below.

\subsection{A critical evaluation of the expressiveness of CTL and ITL}

Let us consider, first, the expressiveness of CTL. In the clinical domains we considered, there are mostly three types of temporal constraints between actions:

(i) sequence, stating that the beginning of an action $a 2$ must follow the end of another action $a 1$. In some cases, the minimum and maximum delays between two sequential actions are specified. Both constraints can be trivially modeled in CTL using the before (or after) and delay primitives;

(ii) concurrence, stating that two actions should start together. This constraint can be trivially modeled in CTL imposing the equality of the starting points of the actions; 

“ALL” temporal constraint between such actions in CTL.

Thus, the most commonly used temporal constraints in clinical guidelines can be easily represented in CTL. However, in our application, we also had to face two kinds of constraints we couldn't model in CTL, i.e., disjointness and periodicity constraints.

(iv) disjointness may be used in order to model the fact that two actions can occur in any order, but cannot overlap in time. It is well known that the "before or after" constraints cannot be modeled in an STP-based framework. The extended TCSP framework could be used to this purpose, but complete constraint propagation onto TCSP requires exponential time [Dechter et al., 91];

(v) periodicity is very frequent in the parts of clinical guidelines concerning the therapeutic treatment of patients. In fact, most therapies involve therapeutic actions to be repeated at regular times (e.g., the daily administration of a given drug). In more complex cases, sequences of actions have to be repeated at regular times ${ }^{10}$. Unfortunately, periodic constraints are outside the expressiveness of CTL, and there is no obvious extension of STP-based frameworks to deal with them (independently of the computational complexity of the extended frameworks). Some insights on the problem of dealing with periodicity are reported in the concluding section.

As regards the expressiveness of ITL, we had no problem in encoding the temporal data regarding instances of actions using our language. In fact, in the given applications, instances were mostly exactly located in time (although, in rare cases, also non-precise dates have been used). More important, in our application domain, a simple rule can be used in order to determine correlation relations between instances (see section 5.1).

\subsection{Preliminary experimental results}

The results in table 3 can be interpreted as follows. The first column (GL) indicates the number of clinical guidelines we considered in the experiment (we have drawn six experiments with just one guideline, and then six

\footnotetext{
${ }^{10}$ As an example, let us consider a guideline coping with Non-Hodgkin lymphoma. CHOP is a cyclic combination chemotherapy regimen which may be articulated as follows. In the first day Cyclophosphamide, Adriamycin and Vincristine are given intravenously. Prednisone is administered orally once a day on days 1-5. Then, there is a 16day pause (up to day 21), after which the same treatment cycle is repeated several times (in most cases 6-8 times). Thus, this treatment regimen involves two nested cycles of actions. Assuming that the repetitions are 6 , the external cycle consists of the 6 repetitions of the whole 21-day regimen. Such a regimen consists of 4 concurrent actions (the oral administration of Prednisone - e.g., between 6 am and $8 \mathrm{am}$ - followed by the intravenous administration of Cyclophosphamide, Adriamycin and Vincristine - e.g. between 8 and $10 \mathrm{am}$ ) on day 1, followed by a cyclic action, representing the oral administration of Prednisone for 4 days once a day - e.g., between 6 am and 8 am.
} 
experiments with three guidelines in the CKB). "Classes (points)" indicates the (approximate) number of starting/ending points of classes we took into account in CKB (the number of classes can be simply obtained dividing the number in the "Classes (points)" column by two). "Patients" indicates the number of patients whose temporal data are managed in IKB. We have drawn different experiments with increasing number of patients. "Instances (points)" indicates the approximate number of points (starting and ending points of instances) in IKB. “Time_IP (seconds)" represents the time (expressed in seconds) to deal with these data, in case we could assume that observations are not complete (i.e., we apply “Integrated_Reasoning_IP”). We completed our experiments to deal with the case of complete observations. The column "Hypotheses (points)" indicates the number of starting/ending points of new instances being hypothesized by the reasoning process (in the case of complete knowledge and no future events), and “Time_CP (seconds)” represents the time (expressed in seconds) to deal with these data, assuming that observations are complete (and there is no future event in IKB). All experiments have been run on a 233 Mhz Pentium II PC, under Windows 98.

\begin{tabular}{|lllllll|}
\hline GL & $\begin{array}{l}\text { Classes } \\
\text { (points) }\end{array}$ & Patients & $\begin{array}{l}\text { Instances } \\
\text { (points) }\end{array}$ & $\begin{array}{l}\text { Time_IP } \\
\text { (seconds) }\end{array}$ & $\begin{array}{l}\text { Hypotheses } \\
\text { (points) }\end{array}$ & $\begin{array}{l}\text { Time_CP } \\
\text { (seconds) }\end{array}$ \\
\hline $\mathbf{1}$ & 100 & 6 & 120 & 3 & 40 & 4 \\
$\mathbf{1}$ & 100 & 12 & 240 & 15 & 80 & 21 \\
$\mathbf{1}$ & 100 & 18 & 360 & 54 & 120 & 75 \\
$\mathbf{1}$ & 100 & 24 & 480 & 128 & 160 & 178 \\
$\mathbf{1}$ & 100 & 30 & 600 & 271 & 200 & 378 \\
$\mathbf{3}$ & 300 & 6 & 120 & 20 & 40 & 21 \\
$\mathbf{3}$ & 300 & 12 & 240 & 33 & 80 & 38 \\
$\mathbf{3}$ & 300 & 18 & 360 & 76 & 120 & 87 \\
$\mathbf{3}$ & 300 & 24 & 480 & 145 & 160 & 197 \\
$\mathbf{3}$ & 300 & 30 & 600 & 288 & 200 & 395 \\
\hline
\end{tabular}

Table 3. Experimental results. Times are expressed in seconds, while the size of CKB and IKB is expressed in terms of time points.

\section{Conclusions and Developments}

In planning, workflows, guidelines, protocols and so on, checking whether the temporal constraints in a general plan (protocol, guideline, workflow) are respected by the plan (protocol, guideline, workflow) instantiation is a fundamental task. Such a task involves integrated temporal reasoning considering both the temporal constraints between the classes of events and the (observed) temporal constraints between their instances. The approach in this paper is, to the best of our knowledge, the first one proposing a general-purpose constraint-based knowledge 
server system supporting such a task. To ensure a wider range of applicability to various real-world application domains, our approach is parametric as regards the completeness of observations and the treatment of future events.

A further step towards the development of powerful and general-purpose classes+instances temporal knowledge servers is certainly the extension of the languages we proposed for temporal constraints. In particular, as discussed in section 6.2 above, a main limitation of our current approach is that it cannot deal with periodicity (i.e., with events that repeat at regular times). In order to deal with periodicity in a general (non ad-hoc) way, at least three related issues have to be faced:

(i) devising an expressive and domain-independent language to express user-defined periodicities (e.g., "the first Tuesdays that follow a holiday - for a given Company X”);

(ii) devising an expressive and domain independent language to represent temporal constraints between repeated events, holding at given (user-defined) periodicities (e.g., "Each Monday, a unit of History precedes a unit of Math; each Tuesday, a unit of Math precedes two units of History”), and suitable reasoning techniques operating on it (e.g., to check the consistency of a set of constraints);

(iii) analysing the integration of temporal constraints on instances and temporal constraints on classes when also periodic events (holding at user-defined periodicities - see issue (i) above) and period-dependent constraints between them (see issue (ii) above) are considered.

We considered the issue (i) in two recent papers [Terenziani, 03c; Egidi \& Terenziani, 04] (two surveys of many other approaches to user-defined periodicity are presented in [Bettini \& De Sibi, 99; Tuzhilin \& Clifford, 95]). In [Terenziani, 97] we faced issue (ii), proposing a general-purpose temporal manager dealing with period-dependent temporal constraints between events. In [Terenziani, 03a] we extended such an approach in order to cope also with numeric quantifiers (e.g., "each Tuesday, a unit of Math precedes two units of History”). On the other hand, issue (iii) is still an open problem. In [Terenziani, 00], we discussed some initial solutions. However, the approach in [Terenziani, 00] only covers cases in which periodic events are taken into account at the level of classes, and the instances of events are exactly located in time (i.e., there is a complete observability of instances, and the starting time and the ending time of each instance is known). A treatment of periodicity in the general framework discussed in this paper requires many extensions and subtle issues to be tackled, and is one of the main challenge of our future research activity. 
We also intend to take into account the possibility of extending our approach to cope with disjointness (see section 6.2). However, we still take into account the trade-off between expressiveness and computational complexity, and we are more inclined to add only tractable extensions to our current approach.

\section{References}

[Allen, 83] J.F. Allen. "Maintaining Knowledge about Temporal Intervals", Communications of the ACM, 26(11): 832-843, 1983.

[Allen, 84] J.F. Allen. "Towards a General Theory of Action and Time", Artificial Intelligence, 23(2): 123-154, 1984.

[Allen, 91a] J. Allen. "Time and Time again: the Many Ways to Represent Time", International Journal of Intelligent Systems, 6(4):341-355, 1991.

[Allen, 91b] J. Allen. "Planning as Temporal Reasoning", In Proceedings of Knowledge Representation, pp. 314, 1991.

[Allen \& Hayes, 85] J. Allen, and P. Hayes. "A Commonsense Theory of Time", In Proceedings ninth International Joint Conference on Artificial Intelligence (IJCAI'95), Los Angeles, CA, pp. 528-531, 1985.

[Allen \& Yampratoom, 93] J. Allen, E. Yampratoom, “Performance of Temporal Reasoning systems”, Sigart Bullettin 4(3), 26-29, 1993.

[Bettini et al., 98] C. Bettini, X.S. Wang, and S. Jajodia. "Temporal semantic assumptions and their use in databases”, IEEE Trans. On Knowledge and Data Engineering 10(2), 1998.

[Bettini \& De Sibi, 99] C. Bettini, R. De Sibi. "Symbolic Representation of User-defined Time Granularities", Proc. TIME'99, IEEE Computer Society, 17-28, 1999.

[Brachman \& Schmolze, 85] R. Brachman, and J. Schmolze, "An Overview of the KL-ONE Knowledge Representation System", Cognitive Science, vol. 9, No. 2, pp. 171-216, April-June 1985.

[Brusoni et al., 95] V. Brusoni, L. Console, and P. Terenziani. "On the computational complexity of querying bounds on differences constraints", Artificial Intelligence 74(2):367-379, 1995.

[Brusoni et al., 97] V. Brusoni, L. Console, B. Pernici, P. Terenziani, "LaTeR: Managing Temporal Information Efficiently", IEEE Expert 12(4), 56-64, 1997.

[Calnavese et al., 98] D. Calnavese, M. Lenzerini, and D. Nardi. "Description Logics for Conceptual Data Modeling”, Logics for DataBases and Information Systems, J. Chomicki and G. Saake eds., Kluwer, 1998. 
[Console \& Terenziani, 99] L. Console, and P. Terenziani. "Efficient Processing of Queries and Assertions about Qualitative and Quantitative Temporal Constraints", Computational Intelligence, 15(4), 442-465, November 1999.

[Dechter et al., 91] R. Dechter, I. Meiri, J. Pearl, "Temporal Constraint Networks", Artificial Intelligence 49, 6195, 1991.

[Egidi \& Terenziani, 04] L. Egidi, P. Terenziani, “A lattice of classes of user-defined symbolic periodicities”. Accepted for publication in Proc. TIME 2004.

[Gerevini \& Cristani, 97] A. Gerevini and M. Cristani, “On Finding a Solution in Temporal Constraint Satisfaction Problems”, Proc. IJCAI’97, 1460-1465, 1997.

[IJIS, 91] K.M. Ford, F.D. Anger (Guest Eds.). Special issue on temporal reasoning. International Journal of Intelligent Systems, 6(4-5):337-567, 1991.

[Levesque \& Brachman, 87] H.J. Levesque and R.J. Brachman, "Expressiveness and Tractability in Knowledge Representation and Reasoning", Computational Intelligence 3, 78-93, 1987.

[Meiri, 91] I. Meiri, "Combining Qualitative and Quantitative Constraints in Temporal Reasoning", In Proceedings National Conference on Artificial Intelligence, pp. 260-267, 1991.

[Morris et al., 93] R.A. Morris, W.D. Shoaff, and L. Khatib, "Path Consistency in a Network of Non-convex Intervals", Proc. thirteenth Int'l Joint Conf. on Artificial Intelligence, pp. 655-660, Chambery, France, 1993.

[Nebel, 90] B.Nebel, Reasoning and Revision in Hybrid Representation Systems, LNCS 422, Springer-Verlag, 1990.

[Schmolze \& Mark, 91] J. Schmolze, W.Mark, "The NIKL Experience", Computational Intelligence 6, 48-69, 1991

[Terenziani, 97] P. Terenziani, “Integrating calendar-dates and qualitative temporal constraints in the treatment of periodic events”, IEEE Trans. on Knowledge and Data Engineering 9(5), 1997.

[Terenziani, 00] P. Terenziani, “Integrated Temporal Reasoning with Periodic Events”, Computational Intelligence, 16(2), 210-256, May 2000.

[Terenziani, 03a] P. Terenziani, “Toward a comprehensive treatment of temporal constraints about periodic events”, International Journal of Intelligent Systems, 18(4), 429-468, 2003.

[Terenziani, 03b] P. Terenziani. Reasoning about time. Encyclopedia of Cognitive Science, Macmillan Reference Ltd, 2003. 
[Terenziani, 03c] P. Terenziani. “Symbolic User-defined Periodicity in Temporal Relational Databases”. IEEE Transactions on Knowledge and Data Engineering, 15(2):489-509, 2003.

[Terenziani et al., 00] P. Terenziani, F. Mastromonaco, G. Molino, M. Torchio. Executing clinical guidelines: temporal issues. Proc. Annual Symposium of the American Association of Medical Informatics, Los Angeles, 2000.

[Terenziani et al., 01] Paolo Terenziani, Gianpaolo Molino, Mauro Torchio. A Modular Approach for Representing and Executing Clinical Guidelines. Artificial Intelligence in Medicine 23, 249-276, 2001.

[Tuzhilin \& Clifford, 95] A. Tuzhilin and J. Clifford, "On Periodicity in Temporal Databases", Information Systems, vol. 20, no.8, pp. 619-639, December 1995.

[VanBeek, 91] P. VanBeek, "Temporal Query Processing with Indefinite Information", Artificial Intelligence in Medicine, 3(6), 325-339, 1991.

[VanBeek, 92] Van Beek, Reasoning about qualitative temporal information, Artificial Intelligence 58, 1992.

[Vila, 94] L. Vila. "A Survey on Temporal Reasoning in Artificial Intelligence", AI Communications 7(1):4-28, 1994.

[Vilain et al., 90] M. Vilain, H. Kautz, and P. VanBeek. "Constraint Propagation Algorithms for temporal reasoning: a Revised Report", D.S. Weld, J. deKleer, eds., Readings in Qualitative Reasoning about Physical Systems. Morgan Kaufmann, pp. 373-381, 1990. 